\title{
Long-term behavior of active longitudes for solar X-ray flares
}

\author{
L. Y. Zhang, H. N. Wang, Z. L. Du, Y. M. Cui, and H. He
}

\begin{abstract}
The National Astronomical Observatories, the Chinese Academy of Sciences, A20, Datun Rd., ChaoYang District, Beijing, PR China e-mail: zhangliyun@bao.ac.cn
\end{abstract}

Received 16 February 2007 / Accepted 14 May 2007

\begin{abstract}
Context. Recently, it was found that two persistent active longitudes of sunspots separated by about $180^{\circ}$ existed on the sun by considering a dynamic reference frame inferred from the differential rotation law. The same phenomenon was found in the longitudinal distribution of powerful X-ray flares.

Aims. To study the statistical characteristics of active longitudes of solar activities, we analyzed the data sets of optical flares associated with all the solar X-ray flares $\geq$ C class observed by GOES during the period of 1975 to 2005, of sunspots collected at the Royal Greenwich Observatory, the US Air Force, and NOAA for the same period as X-ray flares.

Methods. The differential rotation law on the Sun is simply described as $\Omega=\Omega_{0}-B \sin ^{2} \varphi$, where $\Omega$ is the angular velocity at a given latitude $\varphi$, while $\Omega_{0}$ is the equatorial angular velocity and $B$ describes the differential rotation rate. Both $\Omega_{0}$ and $B$ are the parameters that need to be determined from observations.

Results. The authors found that there are two active longitudes separated by $180^{\circ}$ for X-ray flares of any class, which have existed for tens of years, that X-ray flares occur more often near the two active longitudes than sunspots do, and that the non-axisymmetry of the longitudinal distribution of X-ray flares increases with the X-ray flare class.

Conclusions. Stronger solar activities occur more preferentially at certain longitudes.
\end{abstract}

Key words. Sun: activity - Sun: flares - Sun: magnetic fields

\section{Introduction}

The longitudinal distribution of sunspots has been studied since the early 20th century. It has been recognized that sunspots tend to cluster in preferred heliographic longitudes on timescales of days or months (e.g., Chidambara 1932; Bumba \& Howard 1965; Balthasar \& Schussler 1984; Wilkinson 1991). The longitudinal distribution of solar flares has been studied since the 1950s. It has been suggested that there exist "active longitudes", which may last for a year or so, even for a solar cycle (e.g. Warwick 1954; Trotter \& Billings 1962; Warwick 1965; Bai 1988, 1992, 1994; Conway \& Matthews 2003). In claiming the existence of active longitudes for both sunspots and flares, previous studies generally assumed that the rotation period of the active longitudes was equal to the Carrington rotation (CR) period (25.38 days sidereal, 27.275 days synodic). However, it is unreasonable to make such an assumption because the Carrington period is not determined by active longitudes.

Subsequently, by taking the rotation period of the Sun as a free parameter, a couple of active longitudes were detected lasting for about a solar cycle (Bai 1987; Ozguc \& Altas 1996). Even a small change of $0.1 \%$ in the rotation period, would, however, result in a substantial drift in longitude in a few years. Therefore, the reality of active longitudes lasting for several years has not been widely accepted, even though the concept has been around for decades (Bai 1988).

Recently, by considering a dynamic reference frame inferred from the differential rotation law, Usoskin et al. (2005) found double active longitudes for sunspots separated by about $180^{\circ}$, which have been lasting for the past 120 years. It was, however, criticized by Pelt et al. $(2005,2006)$ who claimed that the persistent active longitudes was nearly the result of the data processing method used by Usoskin et al. (2005).

By taking the surface differential rotation into account, Zhang et al. (2007) reported that double active longitudes exist for powerful X-ray flares $180^{\circ}$ apart within the last 30 years, which have more significant non-axisymmetry than those derived from the sunspot distribution within the past 120 years (Usoskin et al. 2005). Furthermore, the double active longitudes present the "flip-flop" phenomenon significantly with a period of about 3.5 years, similar to that found by Berdyugina \& Usoskin (2003) in sunspots and by Mursula \& Hiltula (2004) in the heliospheric magnetic field.

In order to study the long-term behavior of active longitudes for X-ray flares, in this paper we extend the data set from X class flares to sunspots and optical flares associated with X-ray flares above class $\mathrm{C}$. We structure this paper as follows: the data description in Sect. 2, the analysis method in Sect. 3, and finally the conclusion and discussion in Sect. 4.

\section{Data base}

The data base for our study includes data for the X-ray flares and what is needed for sunspots. We selected the GOES data for important X-ray flares above $\mathrm{C}$ class during the period of 1975-2005. They were downloaded from the NGDC database (http://www.ngdc.noaa.gov/stp/SOLAR/ftpsolarflares.html\#xray).

Solar active regions are generally regarded as source regions of X-ray and optical flares. This means that the location of Xray flare source regions can be associated with active regions 
Table 1. X-ray flare classification and peak intensity.

\begin{tabular}{ccc}
\hline \hline Class & $\mathrm{W} / \mathrm{m}^{2}$ & $\mathrm{erg} / \mathrm{cm}^{2} / \mathrm{s}$ \\
\hline $\mathrm{C}$ & $10^{-6} \leqslant I \leqslant 10^{-5}$ & $10^{-3} \leqslant I \leqslant 10^{-2}$ \\
$\mathrm{M}$ & $10^{-5} \leqslant I \leqslant 10^{-4}$ & $10^{-2} \leqslant I \leqslant 10^{-1}$ \\
$\mathrm{X}$ & $I \geqslant 10^{-4}$ & $I \geqslant 10^{-1}$ \\
\hline
\end{tabular}

where optical flares take place. Fortunately, the X-ray flares observed by GOES were mostly identified with optical flare observations. The NGDC database provides the locations of optical flares identified to be associated with X-ray flares. We took the central meridian distance of optical flares as that of X-ray flare source regions. And then, we get Carrington longitudes of X-ray flare source regions by translating the central meridian distance into the Carrington coordinates.

Table 1 shows the X-ray flare classification and peak intensity. For convenience we define $\mathrm{X}$-ray flares in $\mathrm{C}, \mathrm{M}$, and $\mathrm{X}$ classes as C-flares, M-flares, and X-flares, respectively. During the period of 1975-2005, there were 23,233 C-flares, 4,411 Mflares, and $398 \mathrm{X}$-flares.

We used the data on sunspot group locations and areas collected at the Royal Greenwich Observatory, the US Air Force, and the National Oceanic and Atmospheric Administration for the period of 1975-2005, which is also downloaded from the NGDC database (http: //www .ngdc . noaa.gov/stp/SOLAR/ ftpsunspotregions. html). For each sunspot group or single spot, we selected only the record of its first occurrence as was done by Usoskin et al. (2005). About 6000 sunspot groups were selected during the period of 1975-2005.

\section{Analysis method}

\subsection{Dynamic reference frame and differential rotation parameters}

We used a dynamic reference frame (Usoskin et al. 2005) inferred from the differential rotation law on the Sun, described simply as follows,

$\Omega=\Omega_{0}-B \sin ^{2} \varphi$,

where $\Omega$ (deg/day) is the angular velocity at a given latitude $\varphi$, while $\Omega_{0}$ (deg/day) is the equatorial angular velocity and $B$ (deg/day) describes the differential rotation rate. The parameters $\Omega_{0}$ (sidereal) and $B$ need to be determined from observations. As the time step we take the Carrington rotation period (CR), and then Eq. (1) takes the form

$\Omega_{i}=\Omega_{0}-B \sin ^{2} \varphi_{i}$

where $\Omega_{i}$ (deg/day, sidereal) is the same angular velocity as $\Omega$ in Eq. (1), index for $i$ denotes the $i$ th $\mathrm{CR}$, and $\varphi_{i}$ is the peakintensity, weighted average latitude of X-ray flares during this CR. For CRs with no flare activity, linear interpolation of the mean latitude was used.

The normalized intensity of a $k$ th flare in the $i$ th CR is defined as

$W_{k i}=I_{k i} / \sum_{j} I_{j i}$

where $I_{k i}$ is the observed peak burst intensity of the flare, and the sum is taken over all the flares during the $i$ th $C R$. The dynamic
Table 2. Differential rotation parameters for different indices of solar activity.

\begin{tabular}{ccccc}
\hline \hline & \multicolumn{2}{c}{ Northern } & \multicolumn{2}{c}{ Southern } \\
\hline & $B$ & $\Omega_{0}$ & $B$ & $\Omega_{0}$ \\
\hline Sunspots & 1.54 & 14.65 & 1.89 & 14.80 \\
C-flares & 3.91 & 14.81 & 3.47 & 13.79 \\
M-flares & 3.53 & 14.59 & 1.55 & 14.33 \\
X-flares & 3.50 & 14.93 & 3.93 & 14.50 \\
\hline
\end{tabular}

reference frame describes the longitudinal migration of active regions with respect to the Carrington frame due to the differential rotation,

$\Lambda_{i}=\Lambda_{0}+T_{\mathrm{c}} \sum_{j=N_{0}}^{i}\left(\Omega_{j}-\Omega_{\mathrm{c}}\right)$,

where $\Lambda_{i}$ is the expected active longitude in the $i$ th $\mathrm{CR}, \Lambda_{0}$ the location of the active longitude in the $N_{0}$ th CR (the beginning CR of the data set), $T_{\mathrm{c}} \approx 27.27$ days is the Carrington rotation period, $\Omega_{j}$ (deg/day) is the angular velocity as $\Omega_{i}$ in Eq. (2), and $\Omega_{\mathrm{c}}=360^{\circ} / 25.38$ (deg/day) describes the sidereal angular velocity of Carrington frame. Then, in the new reference frame, the longitude of the $k$ th flare in the $i$ th CR is defined as

$\widetilde{\lambda_{k i}}=\lambda_{k i}-\Lambda_{i}-n 360^{\circ}$

where $\lambda_{k i}$ is the observed longitude of the flare in the Carrington frame, and $n$ is defined to keep the revised longitude $\widetilde{\lambda_{k i}}$ within the range $\left[0^{\circ}, 360^{\circ}\right]$.

The deviation between the corrected longitude and the center of one active longitude $\left(90^{\circ}\right.$ or $\left.270^{\circ}\right)$ is defined as

$\delta_{k i}=\min \left(\left|\widetilde{\lambda_{k i}}-90^{\circ}\right| ; \widetilde{\lambda_{k i}}-270^{\circ} \mid\right)$,

where indices $i$ and $k$ denote the CR number and the flare within this rotation, respectively. The total discrepancy between the model and the data is then defined as

$\epsilon=\frac{1}{N} \sum_{i} \sum_{k} W_{k i} \delta_{k i}^{2}$,

where $N=\sum_{i} \sum_{k} W_{k i}$ is the number of CRs with observed flares. Varying the values of $\Omega_{0}$ and $B$ in Eq. (2), $\Lambda_{0}$ in Eq. (4) we search for a pair of $\Omega_{0}$ and $B$ which minimizes the discrepancy $\epsilon$.

For sunspots, instead of the normalized intensity of the $k$ th flare in the $i$ th CR in Eq. (3), the normalized area of the $k$ th sunspot in the $i$ th $\mathrm{CR}$ is defined as

$W_{k i}=S_{k i} / \sum_{j} S_{j i}$

and the $\varphi_{i}$ in Eq. (2) is defined as the area weighted average latitude of sunspots during this CR. The other equations and parameters for sunspots are the same as those for flares.

Because of the known asymmetry between the northern and southern hemispheres, we analyze the data from the two hemispheres separately. First, we select all the X-ray flares above X class and analyze them in the way shown above. Second, we extend the data to all the X-ray flares above $\mathrm{M}$ class and repeat the processing method, then to all the X-ray flares above $\mathrm{C}$ class. Finally, we apply the method to sunspots. The best fitting parameters $\Omega_{0}$ and $B$ obtained both for X-ray flares and for sunspots are shown in Table 2 for the two hemispheres. Northern 

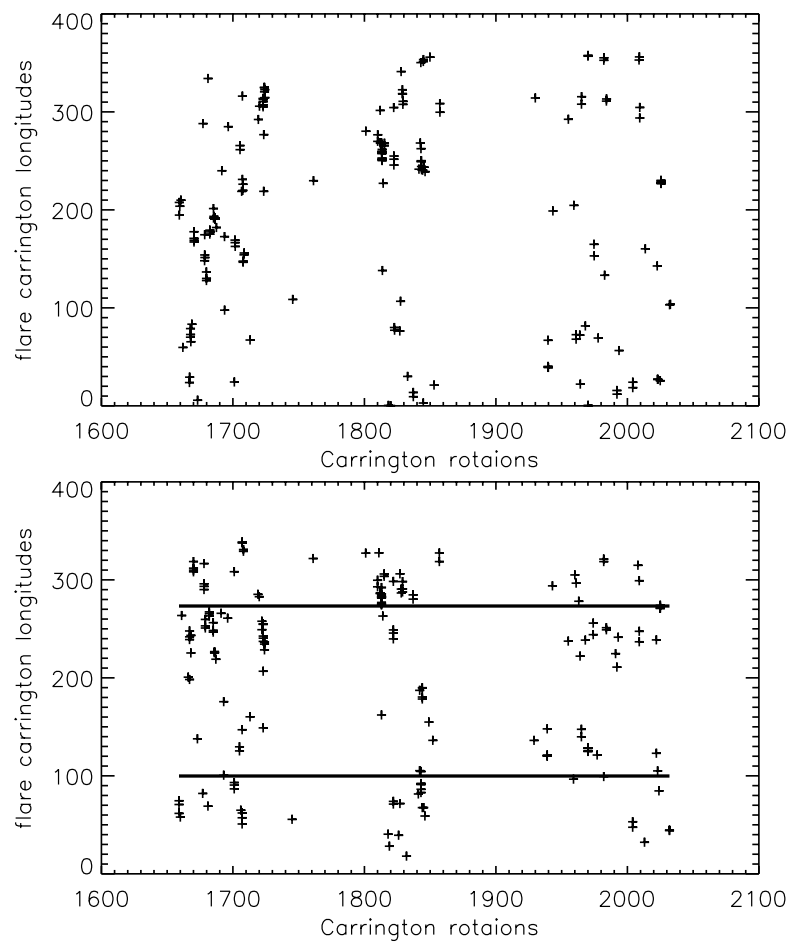

Fig. 1. Upper panel: the observed longitude distribution of X-flares in the Carrington frame for the period of 1975-2005. The horizontal axis denotes Carrington rotations, and the vertical axis the longitudes of $\mathrm{X}$ flares. Lower panel: the revised longitude distribution of X-flares by differential rotation law in the northern hemisphere for the same period as the upper panel. The longitudes for the two horizontal parallel lines are $275^{\circ}$ and $99^{\circ}, 176^{\circ}$ apart, which are the mean longitudes of $\mathrm{X}$-flares that occurred at greater and less than $180^{\circ}$.

and Southern presents the Northern and Southern hemispheres on the Sun. C, M, and X describe the X-ray flare class.

One can see from Table 2 that the parameter $B$ for sunspots in each hemisphere is smaller than that for X-ray flares. This indicates that the differential rotation affects the depth where solar flares occur more significantly than they do where sunspots are formed. Moreover, a group of parameters of $\Omega_{0}$ and $B$ derived from one kind of solar activity differ from those derived from another kind of activity. The uncertainty of $\Omega_{0}$ and $B$ is similar to that of Usoskin et al. (2005). The reasons for that can be explained by the different indices used (different flare class or sunspots) and by the different definitions of the mean latitude and weighting. In addition, the results from different time series (one solar cycle, three cycles, or even longer) are not consistent among themselves either. Therefore, the exact migration mode of the active longitudes needs to be studied further.

\subsection{Longitude-vs.-time distribution}

We can get the revised Carrington longitude $\widetilde{\lambda_{k i}}$ corrected by the differential parameters $\Omega_{0}$ and $B$ according to Eqs. (2), (4), and $(5)$ in the new reference frame. The observed and revised Carrington longitudes of X-flares during 1975-2005 are shown in the upper and lower panels in Fig. 1. The upper panel in Fig. 1 shows the observed occurrence of longitude-vs.-time pattern of $\mathrm{X}$-flares during the latest three solar cycles. There are no X-flares during some CRs in the figure, and most of the X-flares occur around the cycle maxima. But we can see that the X-flares occupy the whole range of Carrington longitudes within the three
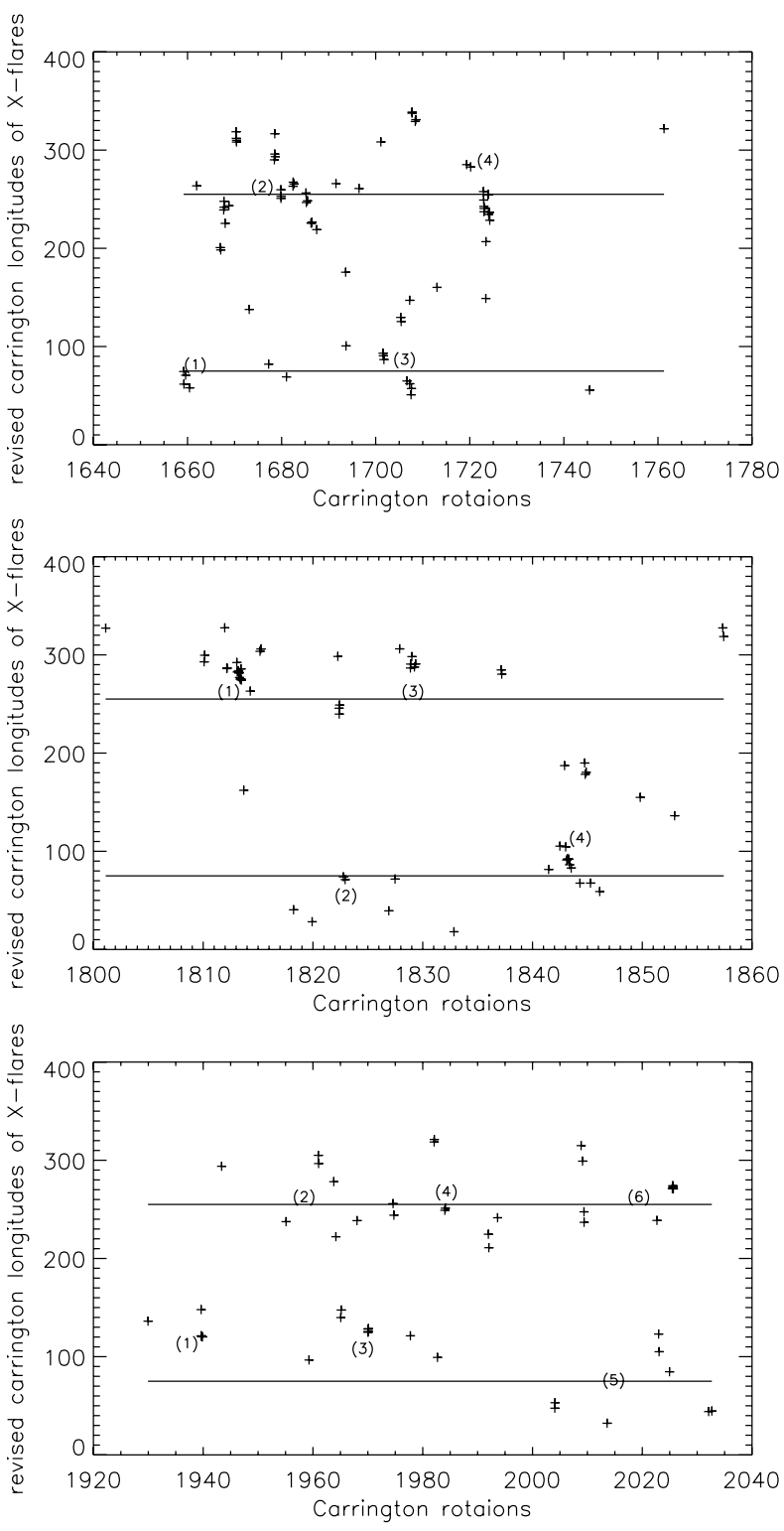

Fig. 2. The enlarged figure of the lower panel of Fig. 1. Upper panel: for the period of 1975-1985 (solar cycle 21). Middle panel: for the period of 1986-1996 (solar cycle 22). Lower panel: for the time of 1997-2005 (solar cycle 23).

cycles. Enlarging the figure, we can see that the flare occurrence migrates from low to high longitudes (see Fig. 2, Zhang et al. 2007) as the solar cycle advances.

Compared with the observed longitude-vs.-time distribution, the revised longitude-vs.-time pattern with the differential rotation law in the lower panel in Fig. 1 presents more clustering. As we can see from the lower panel in Fig. 1, there are two active longitudinal intervals of preferred X-ray flare occurrence centering at around $99^{\circ}$ and $275^{\circ}$ during the period $1975-2005$, in the corrected dynamic reference frame. Therefore, the two active longitudes $180^{\circ}$ apart for X-ray flares do exist for tens of years. But the active longitudes will be smeared by the solar differential rotation effect as time goes on. Enlarging the figure, we can see the "flip-flop" phenomenon according to the alternating to be the dominant one of the two active longitudes (see Fig. 2).

There are four main gathering areas denoted as (1), (2), (3), (4) in the upper panel in Fig. 2, which demonstrates that the 

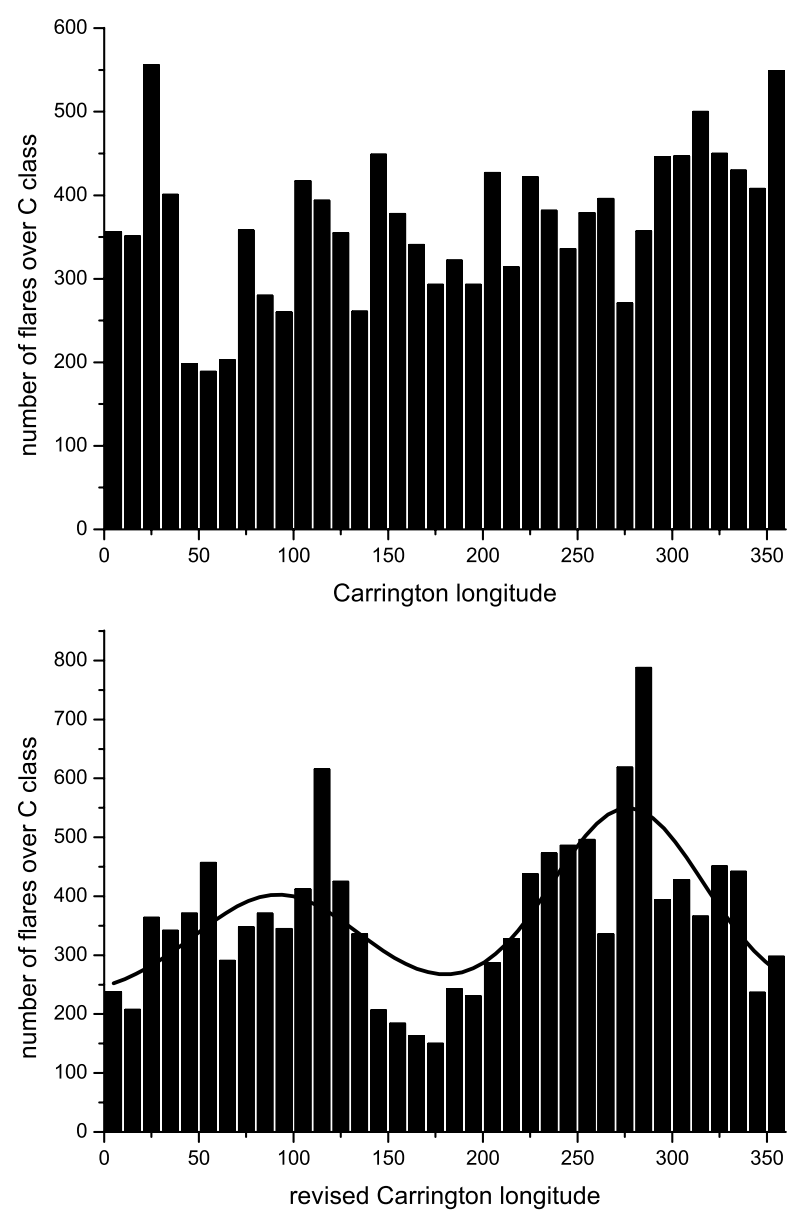

Fig. 3. Upper panel: the observed number distribution of all the flares above the C class in the Carrington frame for the years 1975-2005 in the northern hemisphere. The horizontal axis denotes Carrington longitudes and the vertical axis the number of flares. Lower panel: the same distribution as for the upper panel in the new dynamic reference frame with longitude correction. The solid line depicts the best fit of a double Gaussian. The longitudes of the two peaks of the line are around $90^{\circ}$ and $270^{\circ}$, the longitudes of the two peaks of the histograms are $120^{\circ}$ and $290^{\circ}$. The peaks are almost $180^{\circ}$ apart.

two active longitudes periodically (about 47 CRs or 3.5 years) alternate being the dominant region. In the beginning of the 11-yr cycle, the activity dominates in the lower active longitude, area No. (1). Then it switches up and down several times as the solar cycle advances.

A similar phenomenon can be found in the middle panel in Fig. 2, but the alternation period is about 25 CRs (1.9 years). In the beginning of this cycle the higher active longitude is the dominant one. It again switches, however, down to the lower active longitude during the first several CRs in solar cycle 23. There are six gathering areas in this cycle and the alternation period is $30 \sim 45 \mathrm{CRs}(2.25 \sim 3.36$ years $)$.

\subsection{Number and peak-intensity distribution in longitudes}

We computed the heliographic coordinates of the X-ray flares, using the distance to the central meridian of the accompanying optical flare and recalculating it into Carrington coordinates. Then we divided Carrington longitudes from $0^{\circ}$ to $360^{\circ}$ into 36 boxes, of $10^{\circ}$ each and sum up the number and peak-intensity for each box respectively. Figure 3 shows the observed and
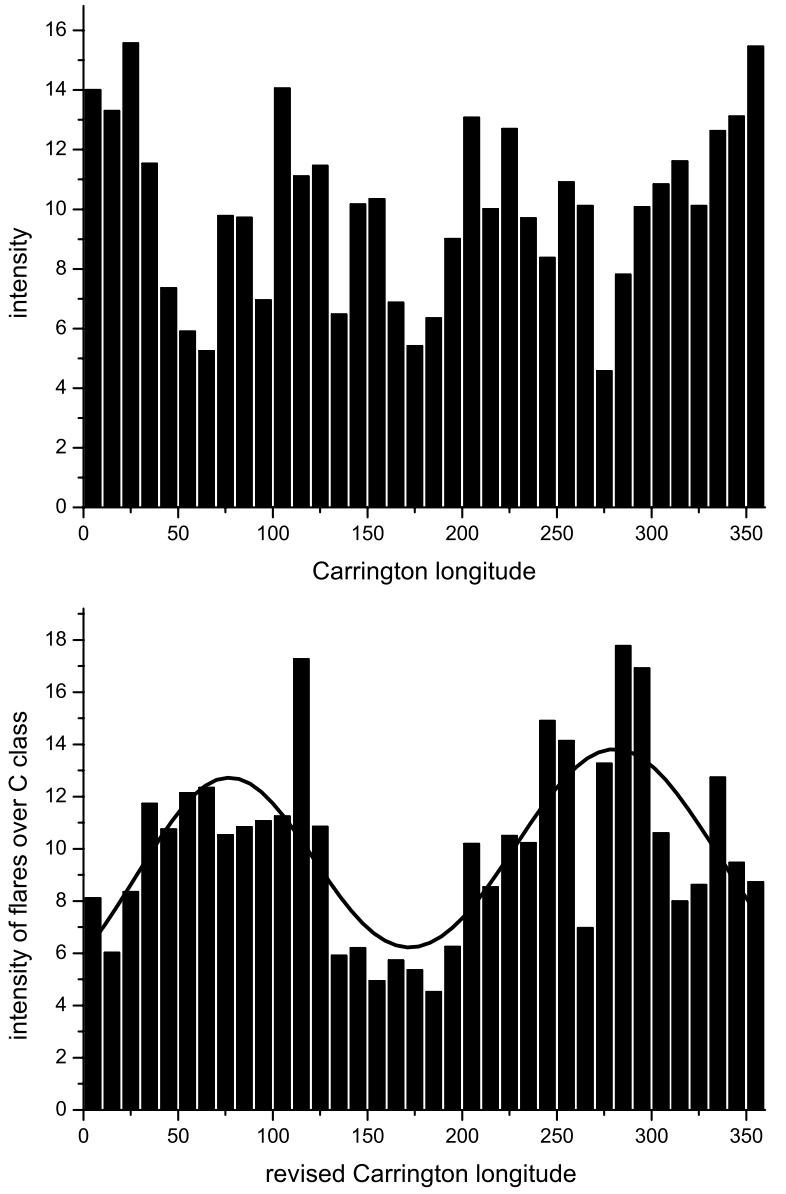

Fig. 4. Upper panel: the observed peak-intensity distribution of all the flares above the $C$ class in the Carrington frame for the years 1975-2005 in the northern hemisphere. The horizontal axis denotes Carrington longitudes and the vertical axis the peak-intensity of flares. Lower panel: the same distribution as in the upper panel in the new dynamic reference frame with longitude correction. The solid line depicts the best fit of a double Gaussian. The longitudes of the two peaks are $90^{\circ}$ and $270^{\circ}$.

revised number distribution of X-ray flares above the $\mathrm{C}$ class in Carrington longitudes during 1975-2005, and Fig. 4 shows the observed and revised peak-intensity distributions of X-ray flares in Carrington longitudes for the same time as the flare number.

The histograms of the observed flare number-vs.-longitude distribution in the upper panel in Fig. 3 show nearly no preferred longitudes for the almost three cycles in the northern hemisphere. The same distribution, but corrected with a differential rotation effect, in the lower panel shows a clear tendency to cluster around the two opposite longitude intervals that centered around $90^{\circ}$ and $270^{\circ}$. The same phenomenon is found in the observed and revised peak-intensity distributions of flares, which are shown in Fig. 4.

The observed peak-intensity distribution of X-ray flares during almost three solar cycles in the Carrington frame (upper panel in Fig. 4) shows no preferred longitudes. But a preference to cluster around two corrected longitudes in the dynamic reference frame is clearly seen in lower panel in Fig. 4, which shows the histogram of the peak-intensity weighted X-ray flare occurrence in the corrected longitude. This is in accord with the earlier result (Berdyugina \& Usoskin 2003) that a signature of the migrating active longitudes is totally smeared out in the Carrington system within 1-2 solar cycles, while a careful account of the 
Table 3. Non-axisymmetry for flare and sunspot numbers.

\begin{tabular}{ccccc}
\hline \hline & \multicolumn{2}{c}{ Northern } & \multicolumn{2}{c}{ Southern } \\
\hline & $\Gamma$ & $N_{1} /\left(N_{1}+N_{2}\right)$ & $\Gamma$ & $N_{1} /\left(N_{1}+N_{2}\right)$ \\
\hline Sunspots & 0.06 & 0.53 & 0.05 & 0.53 \\
C-flares & 0.15 & 0.58 & 0.14 & 0.57 \\
M-flares & 0.27 & 0.63 & 0.20 & 0.60 \\
X-flares & 0.55 & 0.78 & 0.49 & 0.74 \\
\hline
\end{tabular}

$\Gamma$ describes the non-axisymmetry for flare or sunspot number as defined in Eq. (11).

migration of the active longitudes allows us to reveal their persistence.

\subsection{Non-axisymmetry measurement}

To characterize the search results and estimate their significance, we adopted the two kinds of measurements of non-axisymmetry corresponding to the parameters listed in Table 2, according to Zhang et al. (2007). On the one hand, we calculated the nonaxisymmetry for number distribution of X-ray flares as

$N_{1}=\sum_{k, i} 1$, if $\left|\widetilde{\lambda_{k i}}-90^{\circ}\right|<45^{\circ}$ or $\left|\widetilde{\lambda_{\mathrm{ki}}}-270^{\circ}\right|<45^{\circ}$;

otherwise, $N_{2}=\sum_{k, i} 1$,

where $N_{1}$ is the total number of X-ray flares or sunspots that occurred in the two longitude intervals with $45^{\circ}$ distance from the two peak longitudes of $90^{\circ}$ and $270^{\circ}$, and $N_{2}$ is the number of flares or sunspots that occurred outside these two longitude intervals. The non-axisymmetry is defined as

$\Gamma=\frac{N_{1}-N_{2}}{N_{1}+N_{2}}$.

The non-axisymmetery of the number distribution is calculated for C, M, X-flares, and sunspots, respectively, and the results are shown in Table 3 . The percentages of X-ray flares occurred around the active longitudes, i.e. the ratio of $N_{1}$ to $\left(N_{1}+N_{2}\right)$ are also shown there. Northern and Southern represents the Northern and Southern hemispheres of the Sun.

The magnitude of the non-axisymmetry for sunspots is found to be $0.06(0.05)$ for the northern (southern) hemisphere. The magnitude of the non-axisymmetry for X-flares, however, gradually increases from 0.15 (above $\mathrm{C}$ class) to 0.55 (above $\mathrm{X}$ class) in the northern hemisphere which is apparently larger than for sunspots. The same tendency exists in the southern hemisphere.

From Table 3, one can see that the non-axisymmetry for the number of X-flares, which is one of the typical kinds of solar activities, is 0.55 for the northern hemisphere and 0.49 for the southern hemisphere. This implies that $70 \sim 80 \%$ of the powerful X-ray flares occurred around the two active longitudes. Therefore, we can conclude that solar activities do occur at the double preferred longitudes in the Sun for tens of years and that the non-axisymmetry will increase with the importance of solar activities. On the other hand, we calculated the non-axisymmetry for peak-intensity distribution of X-ray flares and for the area distribution of sunspots defined as in Usoskin et al. (2005).

We adopted the following equations

$N_{1}=\sum_{k, i} W_{k i}$, if $\left|\widetilde{\lambda_{k i}}-90^{\circ}\right|<45^{\circ}$ or $\left|\widetilde{\lambda_{\mathrm{ki}}}-270^{\circ}\right|<45^{\circ}$;
Table 4. Non-axisymmetry for flare peak-intensity and sunspot area.

\begin{tabular}{ccccc}
\hline & \multicolumn{2}{c}{ Northern } & \multicolumn{2}{c}{ Southern } \\
\hline & $\Gamma$ & $N_{1} /\left(N_{1}+N_{2}\right)$ & $\Gamma$ & $N_{1} /\left(N_{1}+N_{2}\right)$ \\
\hline Sunspots & 0.12 & 0.56 & 0.09 & 0.54 \\
C-flares & 0.18 & 0.59 & 0.19 & 0.59 \\
M-flares & 0.22 & 0.61 & 0.21 & 0.60 \\
X-flares & 0.42 & 0.71 & 0.43 & 0.72 \\
\hline
\end{tabular}

$\Gamma$ describes the non-axisymmetry for flare peak-intensity or sunspot area.

otherwise, $N_{2}=\sum_{k, i} W_{k i}$

where $N_{1}$ is the total peak-intensity weight of X-ray flares as defined in Eq. (3) or the total area weight of sunspots as defined in Eq. (8), which occurred in the two longitude intervals with $45^{\circ}$ distance from the two peak longitudes of $90^{\circ}$ and $270^{\circ}, \mathrm{N}_{2}$ the total weight of X-ray flares or sunspots that occurred outside these two longitude intervals. The non-axisymmetry $\Gamma$ for flare peak-intensity or sunspot area is defined the same as that for flare numbers (see Eq. (11)). The non-axisymmetry for flare peak-intensity and sunspot area distributions are calculated, too. The non-axisymmetry $\Gamma$ and the ratio of $N_{1}$ to $\left(N_{1}+N_{2}\right)$ are shown in Table 4. Northern and Southern presents the Northern and Southern hemispheres of the Sun.

The magnitude of the non-axisymmetry for the sunspot area is found to be $0.12(0.09)$ for the northern (southern) hemisphere, which is similar to the value 0.11 found by Usoskin et al. (2005). The magnitude of the non-axisymmetry for the peakintensity of X-flares, however, gradually increases from 0.18 (above $\mathrm{C}$ class) to 0.42 (above $\mathrm{X}$ class) in the northern hemisphere which is larger than that for sunspots. The same tendency exists in the southern hemisphere. The non-axisymmetry for the peak-intensity of X-flares to be 0.42 for the northern hemisphere and 0.43 for the southern hemisphere. This corresponds to the percentage of powerful X-ray flares that occurred around the active longitudes, which is more than $70 \%$.

All of this confirms the conclusion obtained previously from the number distribution that solar activities do occur at some preferred longitudes in the Sun for tens of years and that the nonaxisymmetry increases with the grade of solar activity.

\section{Conclusion and discussion}

After applying the surface differential rotation model in the Sun, we analyzed the longitudinal distributions of X-ray flares and sunspots for the latest three solar cycles without any filtering of the raw data. The following conclusions have been reached.

1. By employing the non-axisymmetry and differential rotation model (Usoskin et al. 2005) for sunspots of the latest three solar cycles, the non-axisymmetry of sunspots is proved to be $0.12(0.09)$ in the northern (southern) hemisphere, close to the result (0.11) by Usoskin et al. (2005).

2. Applying the dynamic reference frame to $\mathrm{X}$-ray flares, double active longitudes separated by $180^{\circ}$ in $\mathrm{C}, \mathrm{M}$, and $\mathrm{X}$ classes are found to have existed for thirty years.

3. The "flip-flop" phenomenon exists in every solar cycle with the "flip-flop" period from 1.9 to 3.5 years, in agreement with earlier results for sunspots (Berdyugina \& Usoskin 2003) and heliospheric magnetic field (Mursula \& Hiltula 2004). 
4. The non-axisymmetriey in longitudinal distributions of the flare number and peak flare intensity increases with an increment of X-ray flare importance in both the northern and southern hemispheres, which is more significant than what has been derived from sunspot distributions. This fact implies that stronger solar activity manifestations tend to occur more at active longitudes. In particular, more than $70 \%$ $\mathrm{X}$-flares occurred in the double active longitudes in the last three cycles.

A group of parameters derived from one kind of solar activity does not agree with that derived from another kind of activity. Furthermore, these parameters are also different from those derived from different time series of the observational data. These uncertainties might be caused by the instability of solar differential rotation, from which all models employing the dynamic reference frame are suffered.

The uncertainties on the differential rotation parameters in the present work look consistent with Usoskin et al. (2005). For relatively short time scales, e.g., a year or several months, it is easy to find that solar activities are prominent in some longitude bands (Dodson \& Hedeman 1975). It becomes difficult, however, to reveal the migration rules of the active longitude bands for long time scales, e.g., a solar cycle or even longer, due to the instability of the surface differential rotation in the Sun. Therefore, enhanced research should be done in the future.

In a theoretical sense, the non-axisymmetriy of solar activities implies the non-axisymmetriy existing in the magnetic field in the Sun, which provides new constraints for the development of solar dynamo models as was recently undertaken by Moss (1999, 2004) and Berdyugina et al. (2006).
Acknowledgements. This work is supported by Chinese Academy of Sciences Projects KJCX2-YW-T04 and KGCX3-SYW-403-10, National Ministry of Science and Technology grant No. 2006CB806307, and National Natural Science Foundation of China (NSFC) grants 10673017 and 10233050. We thank Ilya Usoskin for important and useful discussions and the referee for useful suggestions on improving this manuscript. We also thank the GOES and NGDC web teams for data.

\section{References}

Bai, T. 1987, ApJ, 314, 795

Bai, T. 1988, ApJ, 328, 860

Bai, T. 1992, ApJ, 388, L69

Bai, T. 1994, Sol. Phys., 150, 385

Balthasar, H., \& Schussler, M. 1984, Sol. Phys., 93, 177

Berdyugina, S. V., \& Usoskin, I. G. 2003, A\&A, 405, 1121

Berdyugina, S. V., Moss, D., Sokoloff, D., \& Usoskin, I. G. 2006, A\&A, 445, 703

Bumba, V., \& Howard, R 1965, ApJ, 141, 1492

Chidambara, A. 1932, MNRAS, 93, 150

Conway, A. J., \& Matthews, S. A 2003, A\&A, 401, 1151

Dodson, H. W., \& Hedeman, E. R. 1975, Sol. Phys., 42, 121

Moss, D. 1999, MNRAS, 306, 300

Moss, D. 2004, MNRAS, 352, L17

Mursula, K., \& Hiltula, T. 2004, Sol. Phys., 224, 133

Ozguc, A., \& Altas, 1996, Sol. Phys., 163, 327

Pelt, J., Tuominen, I., \& Brooke, J. 2005, A\&A, 429, 1093

Pelt, J., Brooke, J. M., Korpi, M. j., \& Tuominen, I. 2006, A\&A, 460, 875

Trotter, D. E., \& Billings, D. E. 1962, ApJ, 136, 1140

Usoskin, I. G., Berdyugina, S. V., \& Poutanen, J. 2005 A\&A, 441, 347

Warwick, C. S. 1954, ApJ, 120, 237

Warwick, C. S. 1965, ApJ, 141, 500

Wilkinson, L. K. 1991, BAAS, 23, 1030

Zhang, L. Y., Cui, Y. M., Du, Z. L., \& Wang, H. N. 2007, Adv. Space Res., in press 